\title{
Very short term recognition memory for odors
}

\author{
C. JEHL, J.-P. ROYET, and A. HOLLEY \\ Université Claude-Bernard, Villeurbanne, France
}

\begin{abstract}
An investigation of very short term olfactory recognition memory was made with odors of low familiarity to subjects. The experimental procedure was that currently used to make qualitative similarity judgments on odors delivered in paired succession. Subjects made similarity judgments in a yes/no recognition paradigm on odors that were either identical or different. The dependence of recognition performance upon the degree of qualitative similarity was assessed by using two sets of dissimilar odor pairs: slightly dissimilar pairs (S1) and very dissimilar pairs (S2). Performance in terms of correct judgments (hits, correct rejections) was rather good for identical pairs in both sets and was nearly perfect for very dissimilar pairs with a delay of 2-300 sec, suggesting no effect of time or similarity on performance. However, for slightly dissimilar pairs, false alarms increased in number, thereby indicating a dependence of the recognition score on the qualitative distance between odors. In addition, false alarms tended to increase with the lengthening of the retention interval. It was suggested that the subjects based their responses on their capability to detect differences between odors rather than recognizing their similarities. Correct identifications were thus preserved at the cost of increasing false alarms when the discrimination task was made more difficult by closer similarity between odors (S1) or by the fading of memory traces with time. Studying the congruence between the similarity judgments and the kind of evocations associated with paired odors gives some support to the view that recognition performances had some cognitive/semantic basis.
\end{abstract}

In an attempt to examine whether memory for odors displays original properties when compared with memory for other sensory events, its forgetting function has been investigated in several studies using different retention intervals. It appeared that, contrary to what is known about visual memories (Shepard, 1967), the olfactory modality is characterized by a slow rate of forgetting (Engen \& Ross, 1973). However, the forgetting function for odors has most often been explored with relatively long retention intervals (longer than $10 \mathrm{~min}$ ), and little is known about retention during the first $60 \mathrm{sec}$ following initial odor presentation. The only available studies on very short term olfactory memory are those published by Engen, Kuisma, and Eimas (1973), and Mair, Capra, McEntee, and Engen (1980). These authors did not find any variations in correct and incorrect recognition rates under the influence of time. Thus, they presented the relatively flat forgetting function obtained in their study as evidence that odor recognition is only slightly affected by the length of the retention interval. Another characteristic of memory for odors, disclosed in short-term-memory experiments, is the relatively low initial acquisition level. For example, the recognition rate for odors has been evaluated to be $70 \%$ (Engen \& Ross, 1973), whereas the recognition score for pictures has been found to be $99.7 \%$ (Shepard, 1967).

Measures of odor retention function were found to be affected by two factors: subjects' familiarity with odor-

Address correspondence to C. Jehl, Université Claude-Bernard, Laboratoire de Physiologie Neurosensorielle, 43, Bd du 11 novembre 1918, 69622 Villeurbanne Cedex, France. ants and qualitative similarity between the to-beremembered odors and their distractors. A study by Rabin and Cain (1984) has shown that for highly familiar odorants, the recognition score could reach $96 \%$. According to Cain (1979) and Rabin and Cain, there is a strong relation between odor recognition and rated familiarity. A similar improvement of retention scores has also been obtained through similarity manipulations in chosen odorants. As an example, for odors highly distinct in terms of quality, Lawless and Cain (1975) found, even after $10 \mathrm{~min}$, an excellent recognition memory score of $85 \%$. Similarly, Engen and Ross (1973) found memory performance to depend closely on similarity of target and distractor items in a two-alternative forced-choice test occurring $10 \mathrm{~min}$ after stimulus presentation.

The present experiment was aimed at investigating the role of similarity in very short term recognition memory-that is, with delays from 2 to $300 \mathrm{sec}$. The explored period was extended to $300 \mathrm{sec}$ in order to permit us to relate our results with those of longer term experiments (10 min and more). Conditions were analogous to those classically used for making judgments on odors presented in pairs. Two sets of odor pairs were presented in order to test either slightly dissimilar or very dissimilar odorants.

Because recognition scores may notably vary with odor familiarity, the influence of temporal factor on odor recognition memory was examined in test conditions as homogeneous as possible with regard to this factor. It seemed that this condition of homogeneity could be more surely satisfied with unfamiliar rather than with fa- 
miliar odors. Therefore, odors of pure chemical products were chosen because they were less likely to have been often experienced by the subjects. Moreover, previous findings have clearly demonstrated that odor recognition performance is significantly increased in subjects who are provided with the veridical names of the to-beremembered odors before memory testing (Engen \& Ross, 1973; Eskenazi, Cain, \& Friend, 1986; Lyman \& McDaniel, 1986; Murphy, Cain, Gilmore, \& Skinner, 1991; Walk \& Johns, 1984). However, the experimenters systematically used familiar odors. Therefore, the question of whether subjects base their recognition judgments of unfamiliar odors on verbal labeling was also addressed.

Except for recognition paradigms in which a distracting task (i.e., counting backwards) is imposed during the retention period, subjects cannot be prevented from mentally forming associations to odors and using them as cues for odor retrieving. It could be the case that the distinction between successively delivered odors can be made on a cognitive basis rather than on a perceptual one if only one of the odors gives rise to evocations, or if the odors elicit distinctive evocations. For this reason, after delivery of the second odor in a pair, the subjects were required to orally enounce all evocations elicited by both odors. The aim was to ascertain whether or not the evocations mediated and thus facilitated the similarity judgments.

Table 1

List of Two Sets of Odor Pairs

\begin{tabular}{|c|c|c|}
\hline Pair & First Odor & Second Odor \\
\hline \multicolumn{3}{|c|}{ Set 1} \\
\hline 1. & cumene & cumene \\
\hline 2. & alpha-phenylethanol & alpha-phenylethanol \\
\hline 3. & cyclopentanone & cyclopentanone \\
\hline 4. & dipropyl ketone & dipropyl ketone \\
\hline 5. & 3,3,5-trimethyl cyclohexanone & 3,3,5-trimethyl cyclohexanone \\
\hline 6. & methyl heptyl ketone & methyl heptyl ketone \\
\hline 7. & dextro-camphor & 1,8-cineole \\
\hline & iso-amyl acetate & ethyl-n-butyl ketone \\
\hline 9. & dibutyl ketone & diamyl ketone \\
\hline 10. & anisole & acetophenone \\
\hline 11. & benzaldehyde & nitrobenzene \\
\hline 12. & $d$-limonene & para-cymene \\
\hline \multicolumn{3}{|c|}{ Set 2} \\
\hline 1. & coumarin & coumarin \\
\hline 2. & benzophenone & benzophenone \\
\hline 3. & 1,1,2,2-tetrabromoethane & 1,1,2,2-tetrabromoethane \\
\hline 4. & thymol & thymol \\
\hline 5. & beta-phenylethanol & beta-phenylethanol \\
\hline & fenchyl alcohol & fenchyl alcohol \\
\hline 7. & naphthalene & veratrol \\
\hline & methyl cinnamate & iso-hexenol \\
\hline & alpha-ionone & carvacrol \\
\hline & $n$-butyric acid & $l$-menthone \\
\hline & anis aldehyde & safrole \\
\hline 12. & adamantane & linalool \\
\hline
\end{tabular}

Note-Distractors 7-12 in Set 1 were slightly different from the six targets. Distractors $7-12$ in Set 2 were very different from the six targets.

\begin{tabular}{|c|c|c|}
\hline & $t 1$ & $\mathrm{t} 2$ \\
\hline 1 & c & 0 \\
\hline 2 & 0 & $\mathbf{p}$ \\
\hline 3 & $\mathbf{g}$ & $h$ \\
\hline 4 & k & 1 \\
\hline 5 & $i$ & $\mathbf{j}$ \\
\hline 6 & $\theta$ & e \\
\hline 7 & $\uparrow$ & $f$ \\
\hline 8 & $b$ & $b$ \\
\hline 9 & $q$ & $r$ \\
\hline 10 & $m$ & $\mathrm{n}$ \\
\hline 11 & d & $d$ \\
\hline 12 & $\mathbf{a}$ & $\mathbf{a}$ \\
\hline
\end{tabular}

\begin{tabular}{|c|c|c|}
\hline & \multicolumn{2}{|c|}{ Set 2} \\
\hline & $t 1$ & t2 \\
\hline 1 I & $\overline{m^{\prime}}$ & $n^{\prime}$ \\
\hline 2 & $b^{\prime}$ & $b^{\prime}$ \\
\hline 3 & $a^{\prime}$ & $a^{\prime}$ \\
\hline 4 & i' & $\mathbf{j}^{\prime}$ \\
\hline 5 & $k^{\prime}$ & P \\
\hline 6 & $\theta^{\prime}$ & $e^{\prime}$ \\
\hline 7 & $0^{\prime}$ & $p^{\prime}$ \\
\hline 8 & $c^{\prime}$ & $c^{\prime}$ \\
\hline 9 & $d^{\prime}$ & $d^{\prime}$ \\
\hline 10 & $f^{\prime}$ & $f^{\prime}$ \\
\hline 11 & $g^{\prime}$ & $h^{\prime}$ \\
\hline 12 & $q^{\prime}$ & $r^{\prime}$ \\
\hline
\end{tabular}

Figure 1. Two sets (S1 and S2) of 12 pairs of odors delivered successively at time $t 1$, then $t 2$. In each set, six paired odors were identical and six paired odors were either slightly dissimilar (S1) or very dissimilar (S2).

\section{METHOD}

\section{Subjects}

One hundred and eight subjects ( 54 women and 54 men) participated in the experiments. The mean age of the subjects was $35.12 \pm 13.41$ years, with a range from 17 to 75 years. None of them had had any previous experience with a task requiring them to make similarity judgments on odor pairs. Most of them were students or laboratory researchers, and a few had no connection with the university. Occasional smokers represented about $10 \%$ of this population. They were asked to refrain from smoking for several hours preceding the test. The subjects were paid for their participation.

\section{Stimuli}

The stimulus sample included 36 single aliphatic and aromatic compounds that were selected because they were unfamiliar to the subjects and because they represented a large diversity of odor qualities. The stimuli were supposed to be unfamiliar in the sense that they had a low probability of being encountered by the subjects in their daily lives. They were qualitatively diverse in that they could be described by a large range of olfactory notes (Arctander, 1969): anisic (anisole), balsamic (methyl cinnamate), camphoraceous (3,3,5-trimethyl cyclohexanone), creamy (veratrol), earthy (fenchyl alcohol), ethereal (dipropyl ketone), fruity (iso-amyl acetate, $d$-limonene), floral (alpha-ionone, beta-phenylethanol), gassy (para-cymene), green (cumene, iso-hexenol), herbaceous (carvacrol, coumarin, thymol), metallic (benzophenone), minty (dextro-camphor, $l$-menthone), musty (cyclopentanone), rancid ( $n$-butyric acid), rooty (safrole), tarry (naphthalene), and woody (linalool). The stimuli were distributed in two sets of 12 pairs (Table 1 and Figure 1). In the first set (S1) of 12 pairs (Table 1), the distractors (i.e., the second odors from 7 to 12) were qualitatively only slightly different from the targets (first odor). This qualitative proximity between targets and distractors in a pair was inferred from the fact that they could be currently described by using the same main descriptor or note (Arctander, 1969). As an example, the odor pair benzaldehyde-nitrobenzene is described by 
using the descriptor "bitter almond." Likewise, iso-amyl acetate and ethyl- $n$-butyl ketone have the odor of banana in common. Similarity in terms of chemical structure or nature of functional groups was not of primary concern. In the second set (S2), the distractors were very different from the corresponding targets. The pairs made of identical odors and those made of different ones were equally set out in each set.

For testing purposes, each stimulus was presented for inspection in a $120-\mathrm{ml}$ test flask containing a $10-\mathrm{ml}$ solution of an odorous substance. The solution was obtained by diluting $1 \mathrm{ml}$ or $1 \mathrm{~g}$ of a pure chemical product in $9 \mathrm{ml}$ of a solvent, diethyl phtalate. A homogeneous solution was obtained through agitation of the solution for $10 \mathrm{~min}$. For identical-odor pairs, the contents of the test jars were taken from the same solution. The dark color of the jars' walls did not permit the subjects to visually identify the test substances.

\section{Procedure}

As indicated in Figure 2, there were nine groups of 12 subjects each, and each group was assigned to one of the different retention test intervals: $2,10,20,30,40,50,60,120$, and $300 \mathrm{sec}$. The subjects in these different groups were successively tested with the two sets ( $\mathrm{S} 1$ and $\mathrm{S} 2$ ). The presentation order of $\mathrm{S} 1$ and $\mathrm{S} 2$ was counterbalanced to avoid a bias. A resting pause of $45 \mathrm{~min}$ separated the two sets from each other, which permitted the subjects to recover from sensory and cognitive involvements. The number of women and men was equal in each group. In addition, the distribution of subjects in the nine groups of delay was uniform according to their mean age.

The duration for stimulus inspection was fixed at $4 \mathrm{sec}$. This duration allowed the subjects to perform an average of two sniffs and was considered long enough for permitting them to collect information on odors (Laing, 1983, 1985). The odor pairs of each set were delivered in random order to compensate possible effects of sensory adaptation across pairs and fatigue that could build up at the end of the testing session. After the presentation of each pair, there was an interpair interval of $2 \mathrm{~min}$, in which the subjects emitted their judgments on the qualitative similarity of the two odors and then briefly rested. This duration was thought to be long enough to allow the olfactory epithelium to recover from sensory adaptation (Duchamp-Viret, Duchamp, \& Vigouroux, 1989; Sicard \& Holley, 1984; Van As, Kauer, Menco, \& Köster, 1985).

Before memory testing, the subjects were informed that they would be presented with two sets of 12 pairs of odors. For each pair, the odors could be either "identical" or "different." If the odors were different, they could be "slightly dissimilar" or "very dissimilar" to one another. To clearly define the meaning of these terms, the subjects were provided by the experimenter with the following explanations. They were to respond "identical" when they believed that they had been presented twice with the same odor. For slightly dissimilar odors, the grapefruit and orange odors were cited. For highly dissimilar ones, the lilac and diesel fuel odors were mentioned. The subjects were told to remain silent while smelling an odorant and during the retention period in order to avoid any verbal interference with the experimental task. They were also asked to pay close attention to the test odorants.

The subjects inspected the bottles in a quiet, well-ventilated test room. Paired bottles containing an odorous substance were placed in front of them. On each trial, consisting of presentation of an odor pair, the subjects placed under their nostrils each bottle that the experimenter offered them. Once the time for inspection of an odorant was elapsed-say, $4 \mathrm{sec}$ - they gave it back to the experimenter. Immediately after smelling the second odor of a pair-that is, during the interpair interval--the subjects responded with regard to whether the latter odor was identical to or different from the one that had been delivered first. Finally, they were requested to elaborate all kinds of associations of their own with

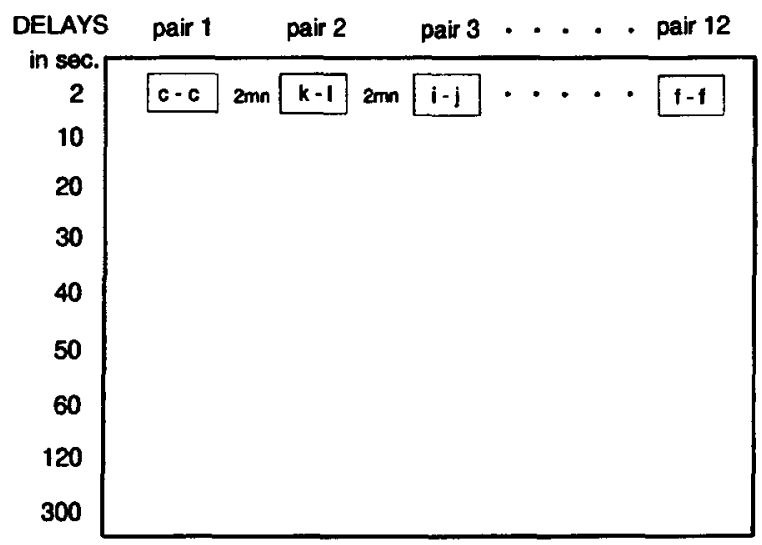

Figure 2. Diagram of the experimental procedure. Twelve pairs of odors, either identical (e.g., $c-c$ or $f-f$ ) or different (e.g., $k-f$ or $i-j$ ), were presented in nine experimental conditions that differed by the delay (from 2 to $300 \mathrm{sec}$ ) between the presentation of two odors of a same pair. The interpair interval was $2 \mathrm{~min}$.

this odor, and the experimenter recorded the responses. The timing of presentation of all pairs of odorants was read on a chronometer.

All evocations provided by the subjects were classified in labeling categories in order to characterize them according to their functional semantic aspects. The labeling categories were (1) the name-for example, lemon, rose, wood, gum; (2) a generic term, such as fruity, flowery, spicy; (3) a non-olfactory sensation, such as heavy, sour, cold, inebriating; (4) a hedonic value, such as pleasant, unpleasant; (5) a life episode of which the odor was reminiscent; and (6) the place where the odor could usually be found, such as hospital, school, laboratory. The place is distinct from the life episode in that, in the former case, the subject's personal experience was not explicitly evoked. When evocations about odors in a pair were not formulated in the same terms, they were taken as distinctive.

\section{RESULTS}

\section{Quantitative Analysis of Discrimination Responses}

From odor similarity judgments, recognition memory performance was assessed by using parameters issued from the signal detection theory (Banks, 1970; Lockhart \& Murdock, 1970). First, judgments led to responses in terms of identity or difference. If the two odors of a pair were identical and were so declared by a subject, a hit was scored. If the odors were incorrectly called "different," a miss was scored. For dissimilar-odor pairs, a correct response of "different" was scored as a "correct rejection," whereas an incorrect response of "identical" was a false alarm. In addition, these variables were transformed into the following parameters: hit rate (HR), false-alarm rate (FR), discrimination measure $\left(d_{\mathrm{L}}^{\prime}\right)$, and bias response $\left(C_{L}\right)$. Calculations, recently described by Snodgrass and Corwin (1988), were performed as follows:

$$
\begin{aligned}
& \mathrm{HR}=\frac{(\text { hits }+0.5)}{(N+1)} \\
& \mathrm{FR}=\frac{\text { (false alarms }+0.5)}{(N+1)}
\end{aligned}
$$




$$
\begin{aligned}
& d_{\mathbf{L}}^{\prime}=\ln \frac{\mathrm{HR}(1-\mathrm{FR})}{\mathrm{FR}(1-\mathrm{HR})} \\
& \mathrm{C}_{\mathrm{L}}=0.5 \ln \frac{(1-\mathrm{FR})(1-\mathrm{HR})}{\mathrm{HR} \cdot \mathrm{FR}}
\end{aligned}
$$

\section{Statistical Analysis}

To analyze the recognition scores, two general statistical methods were used: single and two-way analysis of variance (ANOVA), with or without repeated measurements (Winer, 1962), and multiple orthogonal comparisons, an a posteriori method for establishing the differences between subjects' group means (Dagnelie, 1975). The samples corresponded to the data obtained from each group. In addition, the normality of the samples and the homogeneity of their variance were controlled with the Lilliefors (Conover, 1971) and the Hartley (Winer, 1962) tests, respectively. To analyze the evocation data, the chi-square statistic was used.

\section{Recognition Performance as a Function of Delay}

Figure 3 represents the arithmetic mean of hit scores, false-alarm rates, discrimination measures, and bias responses, respectively, as a function of odor similarity (comparisons between S1 and S2) and as a function of delay.

The two-way ANOVA revealed no reliable difference of hit scores between Sets $\mathrm{S} 1$ and $\mathrm{S} 2[F(1,198)=.07$, n.s.], no effect for delay condition $[F(8,198)=1.58$, n.s.], and no set $\times$ delay interaction $[F(8,198)=.23$, n.s.]. Thus, no significant variation of hit scores was observed for the subjects performing on S1 and S2 as a function of the delay. For false-alarm scores, the two-way ANOVA showed a highly significant difference between Sets $\mathrm{S} 1$ and $\mathrm{S} 2$ in terms of qualitative similarity factor $[F(1,198)=219.75, p<.0005]$, no effect for groups in terms of delay condition $[F(8,198)=1.80$, n.s.], but a significant set $\times$ delay interaction $[F(8,198)=3.39, p<$ $.005]$. The significant effect of qualitative similarity factor is due to the absence of false alarms for the subjects performing on Set S2. Multiple orthogonal comparisons
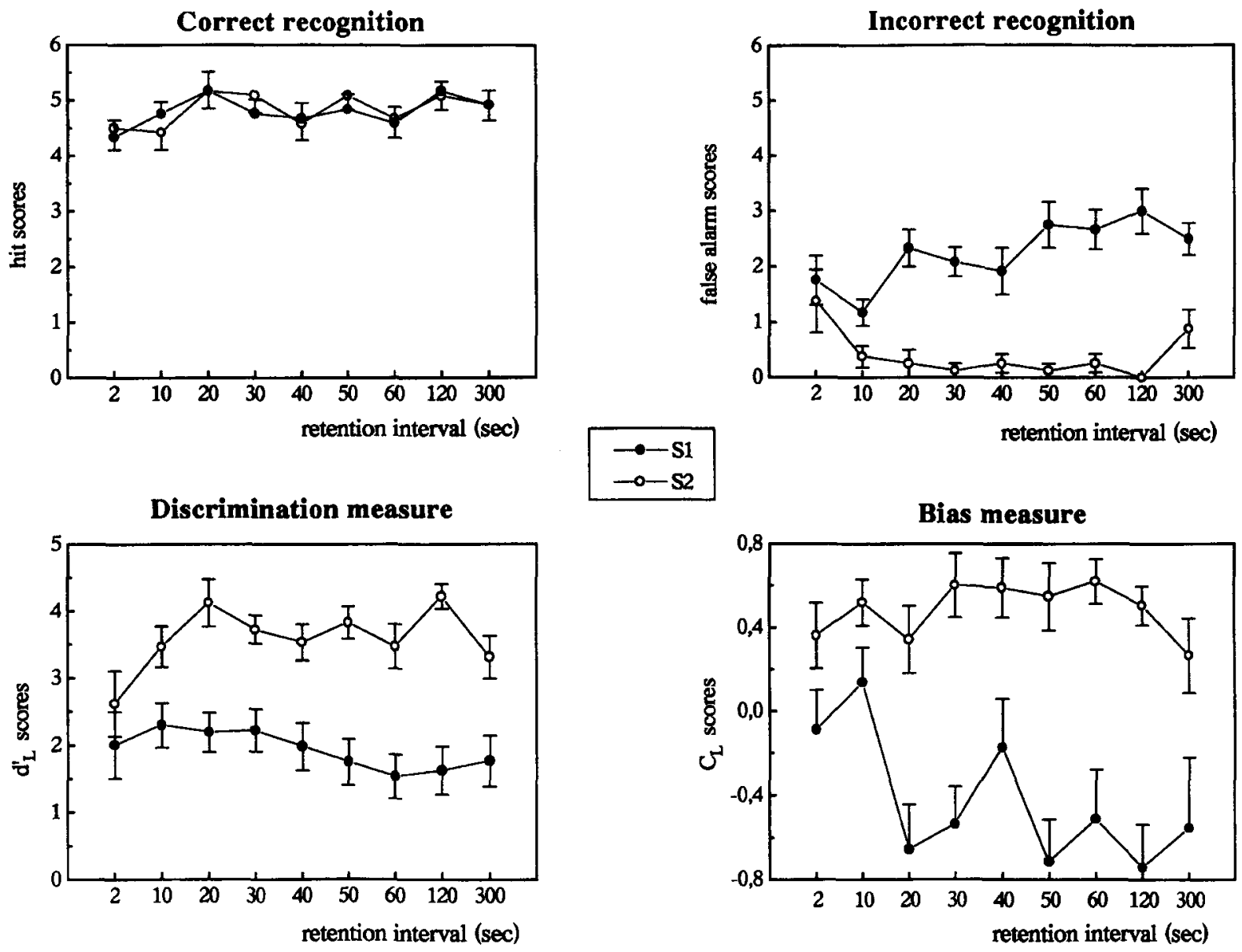

Figure 3. Representation of hit scores, false-alarm scores, discrimination measures, and bias measures as a function of the odor similarity factor (S1 or S2) and as a function of the delay (from 2 to $300 \mathrm{sec}$ ). The vertical bars represent standard errors of the mean. 


\section{Correct recognition}
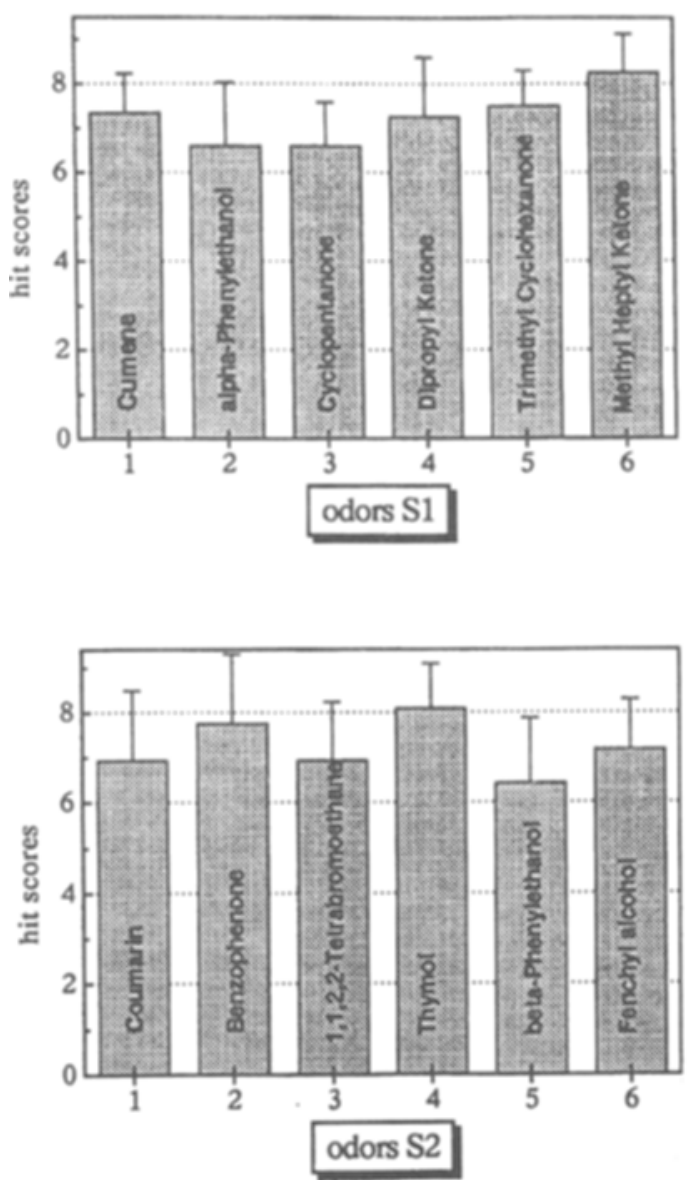

Incorrect recognition
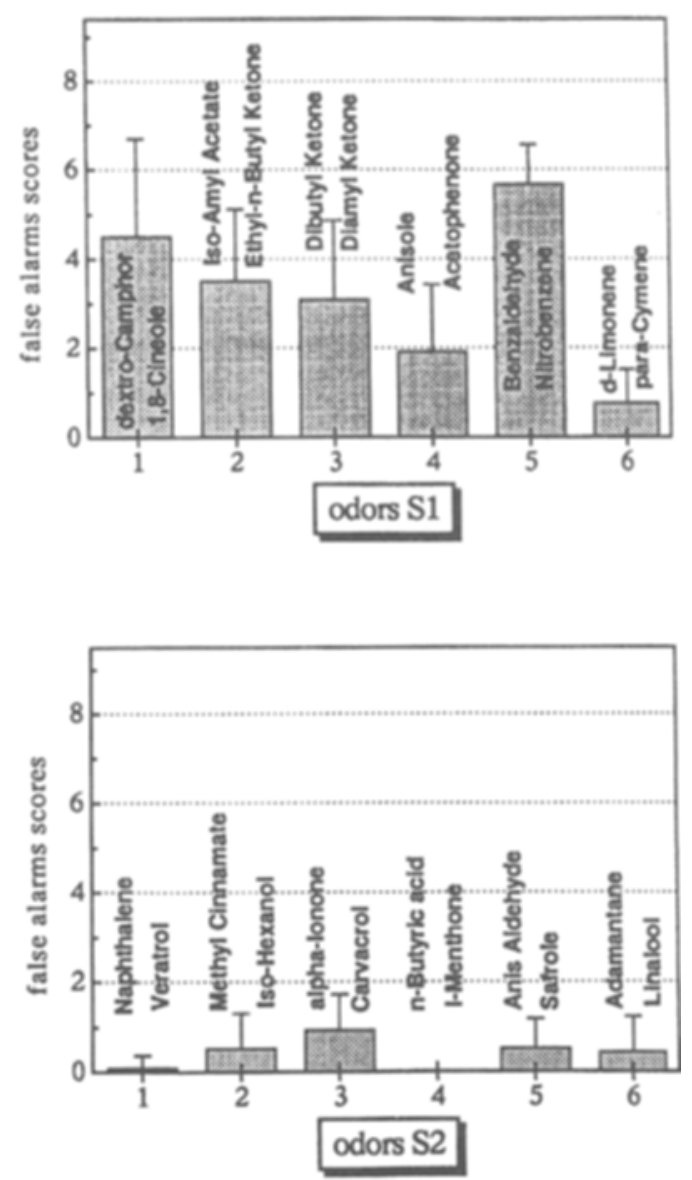

Figure 4. Frequency of correct (hits) and incorrect (false alarms) recognitions for each odor pair from Sets S1 and S2. For correct recognitions (left), the two odors of a pair were identical. The vertical bars represent standard errors of the mean.

between pairs of means show that the false-alarm scores were higher for Set S1 than for Set S2, whatever the delay considered (from 2 to $300 \mathrm{sec}$ ).

For false-alarm scores, the presence of the significant interaction indicates that the effect of delay was different for the two stimulus sets. Thus, the frequency of false alarms seemed to increase with the delay in subjects' performance on S1. A more precise observation of the ANOVA data shows that the estimated value of the statistic $F$ (i.e., $F=1.80$ with 8 and 198 degrees of freedom) approximates the critical value of $F$ for a .05 level of significance (i.e., $F=1.98$ ). The application of a one-way ANOVA on the scores of Set S1 only effectively reveals a significant effect of delay condition $[F(8,99)=$ $2.55, p<.025]$. Indeed, orthogonal comparisons of the means reveal significant increases from 10 to $20 \mathrm{sec}$ and subsequent retention intervals.

For $d_{\mathrm{L}}^{\prime}$ scores, the ANOVA indicated a significantly higher discrimination performance for the subjects performing on Set $\mathrm{S} 2$ than for those on Set $\mathrm{S} 1[F(1,198)=$ $107.99, p<.0005]$. Neither the effect of delay on $d_{\mathrm{L}}^{\prime}$ scores $[F(8,198)=1.2$, n.s. $]$ nor the interaction of set and delay factors was significant $[F(8,198)=1.33$, n.s.]. Except for the 2-sec delay, orthogonal comparisons of the means showed that the scores obtained for $\mathrm{S} 2$ were always significantly higher than those described for S1 (from $p<.025$ to $p<.0005$ ). Finally, an analysis of bias measures indicated higher significant scores for $\mathrm{S} 2$ than for $\mathrm{S} 1[F(1,198)=125.25, p<.0005]$, a just significant effect of delay $[F(8,198)=1.96, p=.05]$, and no set $X$ delay interaction $[F(8,198)=1.83$, n.s.]. Multiple orthogonal comparisons of means revealed that the significant effect of delay factor was due mainly to $C_{L}$ scores of S1 that were higher for 2-sec and 10-sec retention intervals than for the other delays (from 20 to $300 \mathrm{sec}$ ). These two S1 scores obtained with very short delays are not significantly different from those obtained in $\mathrm{S} 2[F(1,198)=3.37$, n.s., and $F(1,198)=2.42$, n.s., respectively].

\section{Recognition Performance for Each Type of Odor Pair}

The number of hits and false alarms is illustrated in Figure 4 as a function of S1/S2 sets (first factor) and 
odor pairs (second factor). Scores for each pair represented the means obtained for different delays. A twoway ANOVA was performed with repeated measurements on the second factor (pairs). The results of the ANOVA did not show significant differences between sets S1 and S2 on scores of correct recognition (left panels) $[F(1,22)=.03]$, but we observed a small significant effect of odor pairs $[F(5,110)=2.48, p<.05]$ and a significant set $\times$ odor pair interaction $[F(5,110)=3.92$, $p<.005]$. Orthogonal comparisons showed that the significant effect of the odor pairs factor is mainly due to the lower number of correct recognitions recorded for the alpha-phenylethanol, cyclopentanone, and betaphenylethanol odors than for the other odors.

The number of false alarms (right panels of Figure 4) was significantly higher for $\mathrm{S} 1$ than for $\mathrm{S} 2$ $[F(1,22)=132.53, p<.0005]$, and it varied significantly as a function of odor pairs $[F(5,110)=16.19$, $p<.0005]$. The interaction between these two factors was significant $[F(5,110)=15.11, p<.0005]$. Orthogonal comparisons revealed that significant differences $(p<.05)$ were obtained for all odor pairs from $\mathrm{S} 1$, examined two by two, except when the second odor pair (iso-amyl acetate-ethyl- $n$-butyl ketone) was compared with the third odor pair (dibutyl ketone-diamyl ketone). In S2, only the number of false alarms of the third odor pair (alpha-ionone-carvacrol) was significantly higher than that of the first odor pair (naphthalene-veratrol) and the fourth odor pair ( $n$-butyric acid- $l$-menthone).

\section{Labeling, Evocations, and Discrimination}

All categories of odor-elicited evocations were pooled across all pairs from both sets and combined across all groups of subjects (Figure 5). Statistical comparisons indicate a reliable difference of the frequency of evocations as a function of the labeling category. Naming odors and not reporting evocations (no label) have a no- tably higher frequency than the other categories (generic, hedonic, place, etc.).

To evaluate the semantic strategy of the subjects, the frequency of evocations was computed for $\mathrm{S} 1$ and $\mathrm{S} 2$ according to three criteria: the qualitative similarity of odors in pairs, the similarity judgment of subjects on odor pairs, and the similarity of evocations described by subjects on these pairs. Frequencies of evocations were therefore determined in eight typical conditions for S1 and eight for S2. Odors of a same pair were either identical (Figure 6) or different (Figure 7). In each case, the subjects judged the odors as either identical (ISJ, identical similarity judgment) or different (DSJ, different similarity judgment). Finally, these two conditions were each divided into two cases according to whether the evocations described by the subjects for the two odors of a same pair were identical or different. When a subject failed to report an evocation for one odor of a pair or for both, the data were not taken into account.

To illustrate identical evocations, two examples can be set up from the data according to whether the two odors of a pair were identical or different. In the first case, the pair alpha-ionone-alpha-ionone was twice given the veridical label "violet." In the second case, the label "eucalyptus" was assigned to both substances from the pair dextro-camphor-1,8-cineole. Regarding different evocations, they are illustrated with three examples. The first is that of a pair made of very different odors, anis aldehyde and safrole. They received different kinds of verbal marks, such as "plastic" and "fennel." The second example deals with slightly dissimilar odor pairs. The pair benzaldehyde-nitrobenzene was given labels taken from the "name" and "place" categories as "bitter almond" and "factory," respectively. Also, the paired odors dibutyl ketone-diamyl ketone were given the labels "green apple" and "fermented apple," respectively. Although the label "apple" was applied to both odors, different qualities are suggested. The attributes "green" and "fermented" indi-

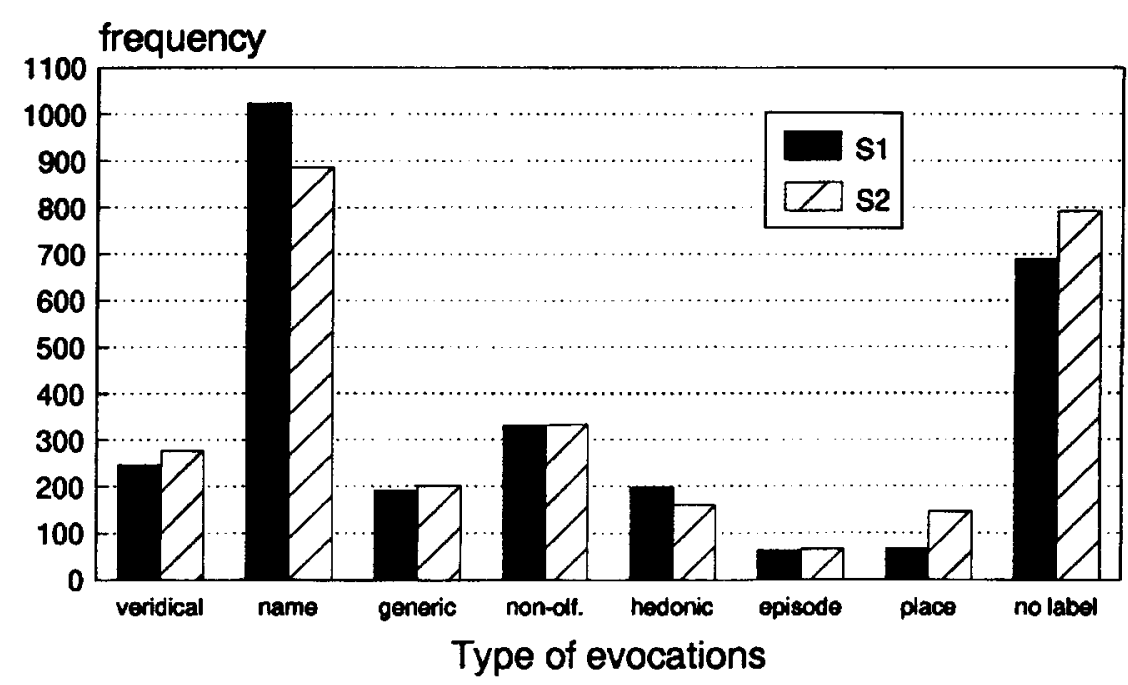

Figure 5. Frequency of evocations provided by subjects for all odor pairs from the two sets, S1 and $\mathbf{S 2}$. 


\section{Pairs of identical odors}

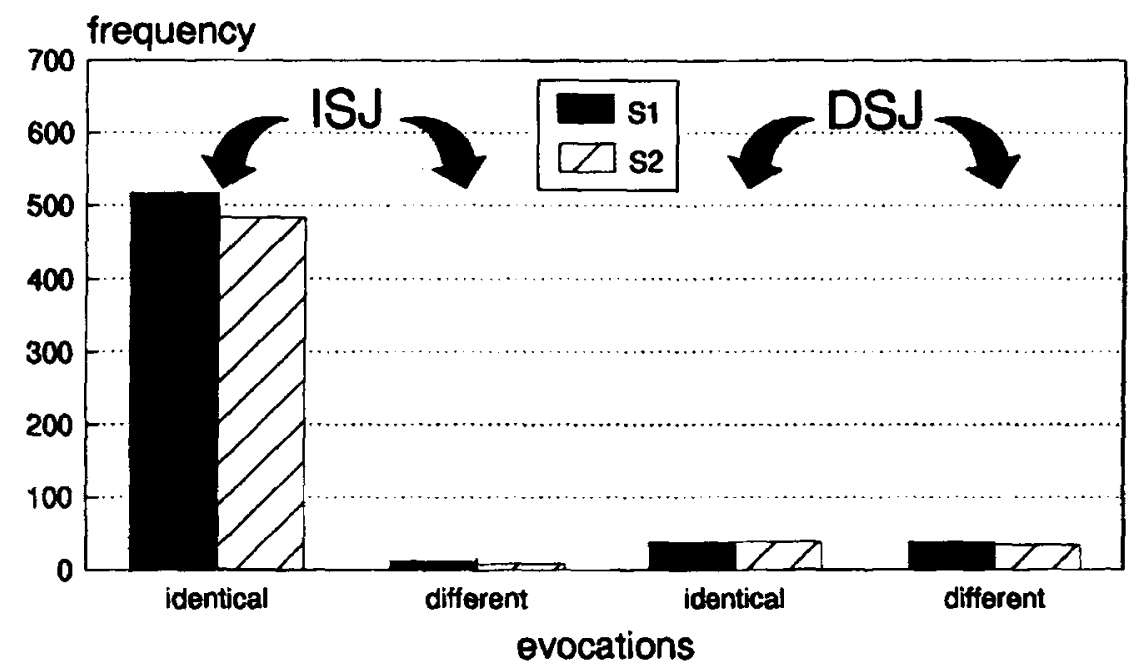

Figure 6. Data from conditions in which the two odors of a same pair were identical. Frequencies of identical and different evocations are depicted when subjects declared the two odors as either identical (ISJ, identical similarity judgment) or difierent (DSJ, different similarity judgment).

cate different maturity stages of the fruit and thereby designate different odor qualities. Finally, the third example illustrates the case of identical odor pairs: The first presentation of beta-phenylethanol was denoted as "rose," whereas the second presentation evoked "printing ink."

When odors of a same pair were identical (Figure 6) and the subjects actually recognized these odors as identical (ISJ), the frequency of identical evocations described by the subjects was much higher than the frequency of different evocations. This holds for $\mathrm{S} 1$ as well as for S2. These results indicate that, when the two odors of a pair are identical, discrimination responses and evo- cations are coherent. When the subjects judged these same odors as different (DSJ), an equal number of identical and different evocations was noted for S1 and S2. Thus, although these scores were small, evocations reported by the subjects were contradictory with respect to their similarity judgments ( $50 \%$ for $\mathrm{S} 1$ and $53.4 \%$ for S2). No significant difference was observed between $\mathrm{S} 1$ and $S 2\left(\chi^{2}=.79\right)$.

When odors of a same pair were different (Figure 7) and the subjects correctly judged these odors as different (DSJ), the frequency of different evocations was relatively lower for S1 than for S2. Correlatively, the fre-

\section{Pairs of different odors}

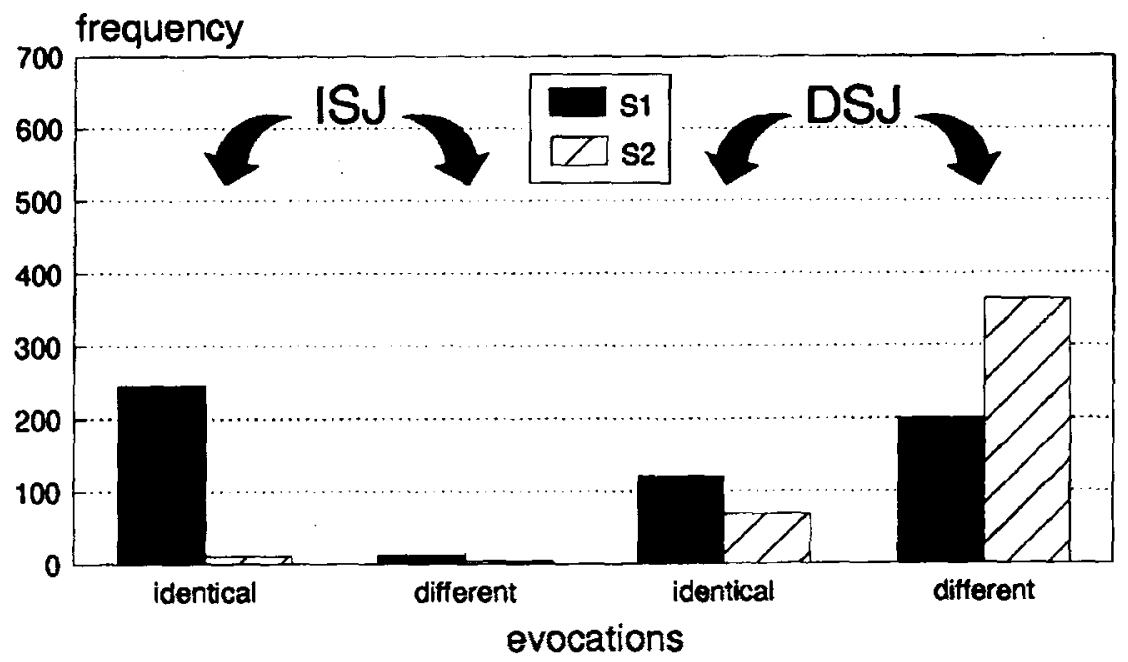

Figure 7. Data from conditions in which the two odors of a same pair were different. Frequencies of identical and different evocations are illustrated when subjects judged the two odors as either identical (ISJ) or different (DSJ). 
Table 2

Frequency of Identical or Different Evocations in Different Conditions

\begin{tabular}{|c|c|c|c|c|c|c|c|c|}
\hline \multirow[b]{4}{*}{ Evocations } & \multicolumn{8}{|c|}{ Odors } \\
\hline & \multicolumn{4}{|c|}{ Set 1} & \multicolumn{4}{|c|}{ Set 2} \\
\hline & \multicolumn{2}{|c|}{ Identical } & \multicolumn{2}{|c|}{ Slightly Dissimilar } & \multicolumn{2}{|c|}{ Identical } & \multicolumn{2}{|c|}{ Very Dissimilar } \\
\hline & ISJ & DSJ & ISJ & DSJ & ISJ & DSJ & ISJ & DSJ \\
\hline $\begin{array}{l}\text { Identical } \\
\text { Different }\end{array}$ & $\begin{array}{c}84.6^{*} \\
3.0\end{array}$ & $\begin{array}{l}6.2 \\
6.2\end{array}$ & $\begin{array}{r}42.5 \\
2.1\end{array}$ & $\begin{array}{l}20.8 \\
34.6^{*}\end{array}$ & $\begin{array}{c}85.7^{*} \\
1.4\end{array}$ & $\begin{array}{l}6.9 \\
6.0\end{array}$ & $\begin{array}{l}2.5 \\
0.9\end{array}$ & $\begin{array}{l}15.2 \\
81.4^{*}\end{array}$ \\
\hline
\end{tabular}

quency of identical evocations was relatively higher for S1 than for S2. Due to the high qualitative similarity of odors, the performance of the S1 subjects was poorer than that of the S2 subjects in terms of congruence between similarity judgments and evocations. When the subjects judged odors as identical (ISJ), and it was scored as a false alarm, identical evocations were observed mainly for $\mathrm{S} 1$. Almost no identical responses were provided for $\mathrm{S} 2$. This result reflects the very low number of false alarms obtained for the $\mathrm{S} 2$ subjects (see Figure 3).

The occurrences of identical and different evocations were calculated in percentage according to the eight conditions considered for S1 and S2 (Table 2). When the two odors of a pair were identical, it was observed that the responses of the subjects (i.e., ISJ as similarity judgment and identical evocations) were correct and consistent for pairs in S1 as well as in S2 $(84.6 \%$ and $85.7 \%$, respectively). When the two odors of a pair were different, the coherence of responses (i.e., DSJ as similarity judgment and different evocations) was observed for $\mathrm{S} 2$ $(81.4 \%)$, but not for S1 (34.6\%). This result must be put together with the high number of errors made by the $\mathrm{S} 1$ subjects and related to the high qualitative similarity of the odors.

\section{DISCUSSION}

\section{Influence of Experimental Factors}

Delay factor. The observed results of correct recognition for pairs of identical odors from both sets (S1 and S2) are consistent with those of previous studies (Engen et al., 1973; Jones, Roberts, \& Holman, 1978; Mair et al., 1980). We indeed did not observe any influence of delay on recognition scores from 2 to $300 \mathrm{sec}$. In the experiment by Engen et al., the proportion of hits tended to be slightly higher than that in the present study, from 3 to $12 \mathrm{sec}$, but the difference was not significant. However, false alarms recorded for dissimilar pairs made apparent the influence of delay. In Set S1, false alarms increased in number with the lengthening of the delay. For very dissimilar pairs from Set S2, no significant difference was observed in the occurrence of false alarms under delay conditions, except for the 2-sec delay. Engen et al. and Mair et al. did not observe any significant effect of the delay on the proportion of false alarms within the first $30 \mathrm{sec}$. This discrepancy with our findings can be due to several factors. First, these authors used sev- eral familiar odors, whereas we used nonfamiliar odors. Second, they investigated the different intervals with the same subjects, and therefore the data were positively correlated. In principle, with such a design, the sensitivity of the experiment should be increased (Winer, 1962). However, each subject performed nearly 100 trials, which resulted in an increased familiarization of the odors and the experimental task as a function of delay. Because the different intervals were tested in a random order, the familiarity effect could be homogeneously distributed across trials, but presumably caused the standard deviation of responses to increase. This procedure could therefore mask significant variations related to delay. Third, the retention period explored by Engen et al. and Mair et al. was shorter than that investigated in the present study ( $30 \mathrm{vs} .300 \mathrm{sec}$ ). In our experiment, the effect of delay on the false-alarm score was not yet significant after $30 \mathrm{sec}$.

To explain why false alarms increased at $2 \mathrm{sec}$, two arguments can be put forward. The first argument is that this delay was so short that sensory adaptation to the target odor was still strong when the distractor odor was delivered (Cain, 1974; Engen, 1982). The second argument is that the delay was not sufficient to allow the first odor from a pair to be entirely encoded when the second odor was presented. As a consequence, the memory processing of the second odor interfered with that of the first odor.

If one disregards the case of the slightly dissimilar pairs-not very well represented in the long-term (10 min) recognition memory experiment of Engen and Ross (1973) - one can compare our data collected for the longest delay studied ( $5 \mathrm{~min}$ ) with Engen and Ross's data with respect to the amount of correct recognitions (hits and correct rejections). Our scores were $80.2 \%$ (hits) for identical pairs from both sets and $90.3 \%$ (correct rejections) for very dissimilar pairs. The scores are relatively high when compared with the correct recognition score of $70 \%$ reported by Engen and Ross. The score reached for pairs of very dissimilar odors is closer to that of $99.7 \%$ reported for the visual modality (Shepard, 1967). One may find the reason in a difference between the paradigms. In our paradigm, the comparison of successively delivered odors required the memorization of a single odor in each trial, whereas in that of Engen and Ross, many odors were delivered before the recognition memory test, which involved a more considerable burden in memory. 
Similarity factor. The false-alarm scores call attention to the sensitivity of the recognition score to the difficulty of the discrimination task. When investigating long-term olfactory memory, Engen and Ross (1973) have also noted an adverse effect of similarity upon recognition scores. Our data are in close accord with the observations of Mair et al. (1980), who assessed olfactory discrimination memory in Korsakoff's patients and in normal observers. These observations indicated that the proportion of correct similarity judgments recorded for identical pairs reached $79 \%$ when they were delivered among slightly dissimilar pairs in one of the experimental sessions and among very dissimilar pairs in the other session (Experiment 2). Their subjects made incorrect judgments with $36 \%$ of false alarms when the pairs were slightly dissimilar and $7 \%$ when the pairs were very dissimilar. In our study, the mean proportions were $80.3 \%, 36 \%$, and $4.5 \%$, respectively. In addition, Figure 4 shows the number of false alarms for each of the six pairs of dissimilar odors evaluated in both sets. Hence, the benzaldehyde-nitrobenzene pair induced the highest proportion of false alarms, suggesting that these odors were more difficult to discriminate than, for example, camphor and cineole, leading to a lower proportion. In this way, the slightly dissimilar pairs can be ordered according to the increasing difficulty of the task, ranging from benzaldehyde-nitrobenzene to limonenecymene. Aside from the latter pair, for which the proportion of false alarms was quite close to that recorded for very dissimilar pairs of $\mathrm{S} 2$, the a priori classification of pairs in two sets, considering the qualitative proximity between their odors, is justified a posteriori. Besides, false alarms reflecting confusions of slightly dissimilar odors can also outline subjects' inability to detect slight differences in quality between odors clustered in a small area of the psychological space (Jones et al., 1978; Lawless \& Cain, 1975), irrespective of delay factor.

\section{Cognitive and Peripheral Processing of Olfactory Information}

Peripheral processing. The experimental task required that the subjects not only remember odors over the course of time, but also discriminate between their qualities. Thus, the kinetics of odor memory cannot be described independently of similarity conditions in which judgments on odors were made. This effect of qualitative proximity must be related to the fact that the recognition test included a discrimination component. Discriminating two stimuli from one another is successful when subjects are able to recognize differential perceptive features in the odors to be compared. It is well known that similarity judgments on paired odors are distributed over a wide range of psychological distances (Engen \& Pfaffman, 1960; Lawless, 1989; Lawless \& Cain, 1975; Lawless, Glatter, \& Hohn, 1991), from close proximity (e.g., benzaldehyde-nitrobenzene) to greater distance (e.g., $n$-butyric acid-mentone). Even though there is no detailed theory of olfactory reception, it is usually assumed that any odorant activates more than one type of receptor (Duchamp, Revial, Holley, \& MacLeod, 1974; Gesteland, Lettvin, \& Pitts, 1965; Gesteland, Lettvin, Pitts, \& Rojas, 1963; Revial, Sicard, Duchamp, \& Holley, 1982), so the degree of perceived similarity between two odorants depends on the degree of overlap of their respective receptors, which is in some complex manner related to the structural similarity between odorant molecules.

Differential abilities of stimuli to adapt their respective receptor sites might influence scores recorded at different retention intervals. Olfactory sensations in humans are classified as rapidly adapting, which means that a loss of sensation accompanies a long-duration sniff or repetitive sniffs of odors at short intervals (Getchell $\&$ Getchell, 1991). In our study, the stimuli were presented for $4 \mathrm{sec}$ and the interstimulus intervals varied from 2 to $300 \mathrm{sec}$. The rate of recovery and the time needed to reach full recovery is dependent on the intensity and the duration of the adapting stimulus. Examples of adaptation to stimuli as short as $4 \mathrm{sec}$ have rarely been reported. Electrophysiological observations suggest that recovery from adaptation occurs on the scale of seconds rather than hours (Moulton, 1971). In humans, Kobal and Hummel (1991) observed that when two brief $(1 \mathrm{sec})$ stimuli were successively presented with a short interval (from 2 to $8 \mathrm{sec}$ ), the EOG response to the second stimulus had a smaller amplitude. However, there was very little overall adaptation of the EOG and no more adaptation after $8 \mathrm{sec}$. Moreover, Cain (1970) found that the magnitude estimates of suprathreshold intensity showed noticeable recovery after only three inhalations of fresh air. The mean duration of an inspiration is $4 \mathrm{sec}$, which is the duration of stimulus presentation in our experiment. Taking into account Cain's observations, subjects could be expected to recover from adaptation after $12 \mathrm{sec}$. Finally, it is currently admitted that cross-adaptation is almost always weaker than self-adaptation (Köster \& De Wijk, 1991). These data allow us to think that, except for $2 \mathrm{sec}$, the progressive variation of the number of false alarms as a function of the retention intervals in the present study cannot be explained in terms of crossadaptation.

Erroneous responses might originate in subjects' basing their discriminating judgments on stimulus intensity rather than on odor quality. Even when the stimuli to be compared were physically strictly similar, it is possible that the memory for subjective intensity declined more rapidly than did the memory for other qualities, leading the subjects to perceive the paired stimuli as different. Barker and Weaver (1983) observed that the same olfactory stimuli presented with a retention interval of 1,5 , or $7 \mathrm{~min}$ are remembered as being substantially weaker the second time than the first one. However, this should have resulted in decreased hit scores, not increased false alarms. Regarding pairing of targets and distractors, strong differences in physical intensity might have provided additional clues, facilitating subjects' rejection of the assumption of similarity and thus reinforcing the basis for a discriminative judgment. However, this effect 
should have resulted not in an increased rate of false alarms, but in an increased rate of incorrect rejections.

Another indication can be drawn from a preliminary test. The subjects were presented with several dilutions $(1 / 10,1 / 100,1 / 1,000)$ of odor pairs from S1 and were asked to match them for subjective intensities. In most cases, they matched stimuli of the same grade, indicating that intensity was not a prevailing factor of odor discrimination.

Cognitive processing. The main feature of bias results is the fact that the false-alarm scores varied under delay and similarity conditions. A possible explanation for this fact is a change in the subjects' decision criterion when judgments were more difficult to make. Reasons might be a priori looked for in methodological aspects, such as instructions given to the subjects, feedback regarding correct responses, and the order of succession between Sets S1 and S2 over the course of the experiment. However, the subjects had no explicit instructions requesting them to adopt a conservative criterion rather than a liberal one, and, conversely, no corrective judgment was provided.

From an ecological point of view, it seems that human beings are more readily able to detect differences in odors to be compared rather than to seek their similarities. The way that the false-alarm scores increased in the presence of the slightly dissimilar pairs can therefore be interpreted as a bias in decision criteria adopted by the subjects, due to the kind of hypotheses they made in order to perform best on the experimental task. They could declare odors from a same pair as identical in quality if they could not find out any feature testifying to their difference. It seems that for slightly dissimilar pairs, the subjects were not able to detect their differences and therefore judged them to be identical. Moreover, delay also produced a bias in decision criteria in making the task more difficult; the subjects could not compare odors of a same pair side by side, but did so according to a mediated fashion, comparing a memorized sensory picture of the first odor with a fresh sensory picture of the second odor.

The study bearing on evocations is useful for seeking a possible strategy used by subjects to aid them to make correct similarity judgments on the pairs from $\mathrm{S} 1$ and S2. This strategy could have been to associate evocations to odors by reference to past olfactory experiences. The collected data show that the number of identical evocations assigned to identical-odor pairs from both sets is far larger than that of different evocations. Thus, a good coherence between the similarity judgment and the nature of evocations is observed for identical pairs.

When the pairs were made of slightly dissimilar odors, the similarity judgments were partially at variance with the nature of the evocations. First, despite a correct judgment- "The odors are different" (DSJ)the subjects provided them with identical evocations. It thus appears that for discriminating odors of low discernibility, the subjects used less conservative criteria when uncertain about their responses, even if they contradicted themselves as they assigned the same evocations to the odors. Second, however, when the subjects incorrectly judged the slightly dissimilar odors as being identical - that is, made false alarms (Figure 7; ISJ)identical evocations were also ascribed to the odors, thereby manifesting coherence between judgments and evocations. However, these same identical evocations (depicted in Figure 7) were only about half as numerous as those attached to pairs of identical odors correctly judged as identical (Figure 6).

The data appear to support the notion that the perceptual basis of the similarity judgments is reinforced by a cognitive process. Realizing that they associate the same evocations to two odors, subjects might infer these odors to be identical and, inversely, infer from their associating different evocations that the odors are dissimilar. Moreover, the results strengthen the idea that the subjects tried to link the odors with verbal labels to use them as mediators for similarity judgments. In previous studies, it has been evidenced that odor recognition memory is enhanced by subjects' ability to correctly identify odors with which they are presented (Murphy et al., 1991; Rabin \& Cain, 1984; Walk \& Johns, 1984). However, it is probable that the high number of identical evocations allocated to the slightly dissimilar pairs expresses a difficulty in perceptually dissociating odors on a qualitative basis. It can also express a difficulty in retrieving distinctive names for very proximate odor sources.

These results indicate limits for the efficiency of verbal labeling on odor discrimination. Furthermore, the low proportion of veridical names used to describe odors confirms that odors had actually been selected on the basis of their low initial familiarity. Besides, the quantitative comparison among evocations of different classes reveals that the subjects referred to nonspecific olfactory features to qualify odors when no name was available. These descriptors were more often connected with taste sensations and esthetic feelings, thereby suggesting connections between the olfactory modality and other sensory modalities.

\section{REFERENCES}

ARCTANDER, S. (1969). Perfume and flavor chemicals. Montclair, NJ: Author.

BANKs, W. P. (1970). Signal detection theory and human memory. Psychological Bulletin, 47, 81-99.

BARKER, L. M., \& Weaver, C. A., III (1983). Rapid, permanent, loss of memory for absolute intensity of taste and smell. Bulletin of the Psychonomic Society, 21, 281-284.

CAIN, W. S. (1970). Odor intensity after self-adaptation and crossadaptation. Perception \& Psychophysics, 7, 271-275.

CAIN, W. S. (1974). Perception of odour intensity and the time-course of olfactory adaptation. ASHRAE Transactions, 80, 53-75.

CAIN, W. S. (1979). To know with the nose: Keys to odor identification. Science, 203, 467-470.

CONOVER, W. J. (1971). Practical nonparametric statistics. New York: Wiley.

DaGnelie, P. (1975). Théorie et méthodes statistiques: Vol. 2. Gembloux, Belgium: Les Presses Agronomiques de Gembloux, A.S.B.L. 
Duchamp, A., Revial, M.-F., Holley, A., \& Macleod, P. (1974). Odor discrimination by frog olfactory receptors. Chemical Senses \& Flavor, 1, 213-233.

Duchamp-Viret, P., Duchamp, A., \& Vigouroux, M. (1989). Amplifying role of convergence in olfactory system: A comparative study of receptor cell and second-order neuron sensitivities. Journal of Neurophysiology, 61, 1085-1094.

ENGEN, T. (1982). The perception of odors. New York: Academic Press.

Engen, T., Kuisma, J. E., \& Eimas, P. (1973). Short-term memory of odors. Journal of Experimental Psychology, 99, 222-225.

Engen, T., \& Pfaffman, C. (1960). Absolute judgments of odor quality. Journal of Experimental Psychology, 59, 214-219.

ENGEN, T., \& Ross, B. (1973). Long-term memory of odors with and without verbal descriptions. Journal of Experimental Psychology, 100, 221-227.

Eskenazi, B., Cain, W. S., \& Friend, K. (1986). Exploration of olfactory aptitude. Bulletin of the Psychonomic Society, 24, 203-206.

Gesteland, R. C., Letrvin, J. Y., \& Pitrs, W. H. (1965). Chemical transmission in the nose of the frog. Journal of Physiology, 181, 525-559.

Gesteland, R. C., Lettvin, J. Y., Pitts, W. H., \& Rojas, A. (1963). Odor specificities of the frog's olfactory receptors. In Y. Zotterman (Ed.), Olfaction and taste I (pp. 19-34). London: Pergamon.

Getchell, T. V., \& Getchell, M. L. (1991). Physiology of olfactory reception and transduction: General principles. In D. G. Laing, R. L. Doty, \& W. Breipohl (Eds.), The human sense of smell (pp. 61-76). Berlin: Springer-Verlag.

Jones, F. N., Roberts, K., \& Holman, E. W. (1978). Similarity judgments and recognition memory for some common spices. Perception \& Psychophysics, 24, 2-6.

Kobal, G., \& Hummel, T. (1991). Human electro-olfactograms and brain responses to olfactory stimulation. In D. G. Laing, R. L. Doty, \& W. Breipohl (Eds.), The human sense of smell (pp. 135-151). Berlin: Springer-Verlag.

Köster, E. P., \& DE WIJK, R. A. (1991). Olfactory adaptation. In D. G. Laing, R. L. Doty, \& W. Breipohl (Eds.), The human sense of smell (pp. 199-215). Berlin: Springer-Verlag.

LAING, D. G. (1983). Natural sniffing gives optimum odour perception for humans. Perception, 12, 99-117.

LAING, D. G. (1985). Optimum perception of odor intensity by humans. Physiology \& Behavior, 34, 569-574.

LAWLESS, H. T. (1989). Exploration of fragrance categories and ambiguous odors using multidimensional scaling and cluster analysis. Chemical Senses, 14, 349-360.
LAWLESS, H. T., \& CAIN, W. S. (1975). Recognition memory for odors. Chemical Senses \& Flavor, 1, 331-337.

Lawless, H. T., Glatter, S., \& HohN, C. (1991), Context-dependent changes in the perception of odor quality. Chemical Senses, 16, 349360 .

LockharT, R. S., \& MURDOCK, B. B. (1970), Memory and the theory of signal detection. Psychological Bulletin, 74, 100-109.

LyMAN, B. J., \& MCDANIEL, M. A. (1986). Effects of encoding strategy on long-term memory for odours. Quarterly Journal of Experimental Psychology, 38A, 753-765.

MaIr, R., CApra, C., McEnTeE, W. J., \& Engen, T. (1980). Odor discrimination and memory in Korsakoff's psychosis. Journal of Experimental Psychology, 6, 445-458.

Moulton, D. G. (1971). The olfactory pigment. In L. M. Beidler (Ed.), Handbook of sensory physiology: IV. Chemical senses (Part 1, pp. 59-74). Berlin: Springer-Verlag.

Murphy, C., Cain, W. S., Gilmore, M. M., \& Skinner, R. B. (1991). Sensory and semantic factors in recognition memory for odors and graphic stimuli: Elderly vs. young persons. American Journal of Psychology, 104, 161-192.

RabiN, M. D., \& CaIN, W. S. (1984). Odor recognition: Familiarity, identifiability, and encoding consistency. Journal of Experimental Psychology: Learning, Memory, \& Cognition, 10, 316-325.

Revial, M.-F., Sicard, G., Duchamp, A., \& Holley, A. (1982). New studies on odour discrimination in the frog's olfactory receptor cells: I. Experimental results. Chemical Senses, 7, 175-190.

SHEPARD, R. N. (1967). Recognition memory for words, sentences and pictures. Journal of Verbal Learning \& Verbal Behavior, 6, 156-163.

SiCARD, G., \& Holley, A. (1984). Receptor cell responses to odorants: Similarities and differences among odorants. Brain Research, 292, 283-296.

Snodgrass, J. G., \& Corwin, J. (1988). Pragmatics of measuring recognition memory: Applications to dementia and amnesia. Journal of Experimental Psychology: General, 117, 34-50.

Van As, W., Kauer, J. S., Menco, B. M., \& Köster, E. P. (1985). Quantitative aspects of the electro-olfactogram in the tiger salamander. Chemical Senses, 10, 1-21.

WALK, H. A., \& JoHNS, E. E. (1984). Interference and facilitation in short-term memory for odors. Perception \& Psychophysics, 36, 508514.

WINER, B. J. (1962). Statistical principles in experimental design. New York: McGraw-Hill.

(Manuscript received April 12, 1993; revision accepted for publication July 7,1994 .) 